\title{
Transarterial chemoembolization using iodized oil for unresectable hepatocellular carcinoma: perspective from multistep hepatocarcinogenesis
}

This article was published in the following Dove Press journal:

Hepatic Medicine: Evidence and Research

3 July 2014

Number of times this article has been viewed

\section{Kengo Yoshimitsu}

Department of Radiology, Faculty of Medicine, Fukuoka University, Fukuoka, Japan
Correspondence: Kengo Yoshimitsu Department of Radiology, Faculty of Medicine, Fukuoka University, 7-45-I

Nanakuma, Jonan-ku,

Fukuoka, 8I4-0I80, Japan

Tel +8192801 I0II

Fax +8I 928646652

Email kengo@fukuoka-u.ac.jp
Abstract: Transarterial chemoembolization (TACE) using iodized oil (Lipiodol ${ }^{\circledR}$ ) (Lp-TACE) as a carrier of chemotherapeutic agents has been routinely performed to control hepatocellular carcinomas (HCC) in Japan, and its use has yielded fairly beneficial therapeutic results. Lipiodol is thought to pass through the tumor sinusoids of HCC and reach the outflow drainage areas, namely, the portal venous side of the tumor. By doing this, Lipiodol blocks not only the tumor's arterial inflow but also its portal venous outflow, providing sufficient ischemic effects. It is known that the inflow blood system, tumor sinusoids, and outflow blood system change drastically during the process of multistep hepatocarcinogenesis; thus, it is reasonable to postulate that the distribution of Lipiodol and the subsequent therapeutic effect of Lp-TACE may also change during that process. Arterial inflow to HCC is highest for moderately differentiated $\mathrm{HCC}(\mathrm{mHCC})$ and is relatively low in well or poorly differentiated $\mathrm{HCC}$ (wHCC and pHCC, respectively). It has been suggested that the metabolic state of $\mathrm{wHCC}$ and $\mathrm{mHCC}$ is aerobic, while that of $\mathrm{pHCC}$ is anaerobic. The tumor sinusoids in wHCC and $\mathrm{mHCC}$ are small in size and large in number, while those in $\mathrm{pHCC}$ are large in size and small in number. This finding results in a greater chance of tumor cell exposure to chemotherapeutic agents in the former and a lesser chance in the latter. The outflow tract, namely, the drainage system via the residual portal venous branches within the pseudocapsule, is more complete in $\mathrm{mHCC}$ and $\mathrm{pHCC}$ and less so in wHCC. Considering all of these components of $\mathrm{HCC}$ of different histological grades, Lp-TACE should have the greatest effect on $\mathrm{mHCC}$ and a relatively low effect on wHCC and pHCC. To achieve consistently high therapeutic results, it is important to consider these components, which affect the sensitivity of HCC to Lp-TACE, to maximize both the chemotherapeutic and ischemic effects of this therapy.

Keywords: liver cancer, multistep carcinogenesis, embolotherapy Lipiodol, histological grade

\section{Introduction}

Hepatocellular carcinomas (HCC) are histopathologically characterized by their abundant vascularity and the presence of a tumor plate/sinusoid system, and these structures and related hemodynamics have been shown to change dramatically during the process of multistep carcinogenesis. ${ }^{1-10}$

Transarterial chemoembolization (TACE) is an accepted treatment method for HCC; however, it is a palliative, rather than curative, therapeutic option. ${ }^{11,12}$ TACE usually involves chemotherapeutic agent injection followed by embolic material administration into the feeding arteries of the HCC. ${ }^{11,12}$ In Japan, TACE has been performed using iodized oil (Lipiodol ${ }^{\circledR}$; Guerbet, Villepinte, France) (Lp-TACE) as a carrier of antitumor agents, and its use has achieved fairly good therapeutic results. ${ }^{11-15}$ The differences in the 
effects of Lp-TACE and TACE without Lipiodol are not fully understood, but are thought to be related to the half-liquid property of Lipiodol. ${ }^{11,12}$ It is thought that Lipiodol can pass through the tumor sinusoids of HCC and reach the outflow drainage areas, namely the portal venous side of the tumor or the areas radiologically appreciated as "corona-like enhancements" on computed tomography (CT) during hepatic arteriography (CTHA). ${ }^{14,15}$ Thus, Lipiodol may block not only the tumor's arterial inflow but also its portal venous outflow. ${ }^{14,15}$

It is known that the inflow blood system, tumor sinusoids, and outflow blood system change dramatically during the process of multistep hepatocarcinogenesis; thus, it is reasonable that the distribution of Lipiodol and the subsequent therapeutic effects of Lp-TACE may also change during that process. In this review article, the presumed mechanism of Lp-TACE is presented, particularly in the context of the structural and hemodynamic changes of HCC during multistep hepatocarcinogenesis.

\section{Hemodynamic and structural changes of HCC during multistep hepatocarcinogenesis Inflow system}

The hemodynamic changes in inflow for HCC during hepatocarcinogenesis have been well-documented ${ }^{1-7}$ and are briefly summarized in Figure 1. During the hepatocarcinogenic state of premalignant low-grade dysplastic nodules, the lesions are supplied by both hepatic arterial and portal venous blood flow in the same manner as normal liver parenchyma; however, when the lesions progress toward more malignant highgrade dysplastic nodules, their arterial supply diminishes, while their portal venous supply is unchanged. When the lesions become early HCC (eHCC), their portal venous flow diminishes in addition to their arterial flow. This causes the lesions to become hypoxic and leads to abundant angiogenesis

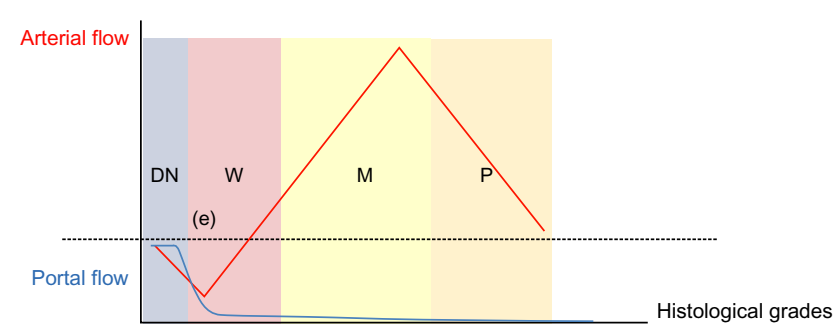

Figure I Hemodynamic changes during multistep hepatocarcinogenesis.

Notes: DN, W, M, and P represent dysplastic nodules, well-differentiated, moderately differentiated, and poorly differentiated hepatocellular carcinomas, respectively; (e) represents early hepatocellular carcinoma, which is a hypovascular, well-differentiated tumor. Note arterial inflow to hepatocellular lesions shows a triphasic pattern, whereas portal venous inflow decreases in a monophasic fashion. or neovascularization. More strictly, it has been shown that neovascularization begins, to some extent, at the stage of dysplastic nodules, ${ }^{2}$ and becomes prominent at the eHCC stage, which corresponds histopathologically to well-differentiated HCC (wHCC). It has also been suggested that two different types of vessels are involved in this neovascularization process. ${ }^{16}$ As neovascularization increases, native arterial and portal venous supply further diminish, and eventually, disappear completely. At that time, the lesions are supplied exclusively by arterial flow via neovascularized vessels (tumor vessels), and the lesions correspond histopathologically to well to moderately differentiated HCC (mHCC). When HCC further dedifferentiates toward poorly differentiated $\mathrm{HCC}$ ( $\mathrm{pHCC}$ ), the arterial supply becomes diminished compared to mHCC. ${ }^{6,7}$ Thus, findings of HCC nodules on the arterial phase of dynamic CT or magnetic resonance imaging (MRI) may change in a triphasic fashion during the process of hepatocarcinogenesis as follows: no or little enhancement in dysplastic-to-early HCC nodules, subtle or weak enhancement in wHCC nodules, robust or prominent enhancement in $\mathrm{mHCC}$ nodules, and again subtle or weak enhancement in $\mathrm{pHCC}$ nodules (Figure 1). It has been reported that well, moderately, and poorly differentiated HCC comprise approximately $15 \%$, $65 \%$, and $20 \%$ of surgically resected lesions, respectively. ${ }^{7}$

Previous investigations have suggested that anaerobic metabolism (glycolysis) predominates in pHCC, while aerobic metabolism predominates in ${ }^{\mathrm{HCC}}$ to $\mathrm{mHCC},{ }^{7}$ which may be the result of the decreased number of tumor vessels in $\mathrm{pHCC}$. In other words, HCC address hypoxic stress by increasing the number of tumor vessels during the wHCC to $\mathrm{mHCC}$ transition and by shifting its metabolism from an aerobic to anaerobic state during the $\mathrm{mHCC}$ to $\mathrm{pHCC}$ transition.

\section{Sinusoidal structure}

One of the histopathologic characteristics of HCC is the presence of tumor plates and tumor sinusoids, which imitate normal liver tissue. It has been well-documented that as the dedifferentiation of HCC progresses, the tumor plates tend to become thicker and more irregular; in other words, in wHCC, the tumor plates are thin and regular in shape, while in pHCC, they are thick and irregular (Figure 2). ${ }^{17,18}$ The tumor sinusoids are the vascular space between the tumor plates that convey the bloodstream and contrast medium, and their structure is affected in a sequential manner during multistep hepatocarcinogenesis. ${ }^{8}$ We have previously shown that the total area of the tumor sinusoids is constant irrespective of HCC histological grade; thus, the tumor sinusoids are smaller in size but larger in number during the wHCC state and 


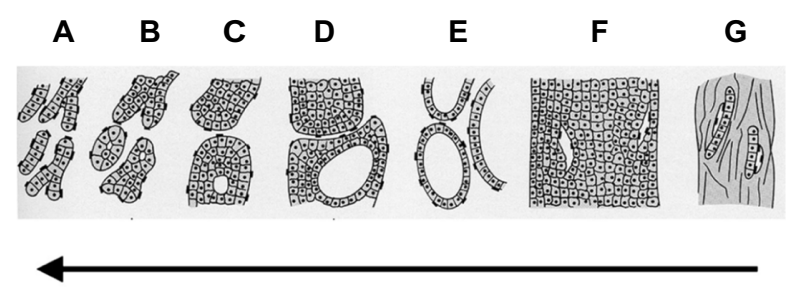

Completion of tumor plate and tumor sinusoid

Figure 2 Schematic presentation of architectural subtypes of hepatocellular carcinoma originally described by the Liver Cancer Study Group of Japan.

Notes: (A) pure trabecular type; (B) trabecular type with small acinar structure; (C) trabecular type with large acinar structure; (D) trabecular type with pseudoglandular structure; (E) pseudoglandular type; (F) compact type; and (G) scirrhous type. Percentage of tumor plate completion and tumor sinusoid number increases in the direction of the arrow, along with the degree of histological differentiation. Copyright (C) 99I. Bunkodo Co. Ltd. Reproduced from Okudaira M. II kansaibougan 2. soshiki bunrui [Hepatocellular carcinoma 2. Histological classification]. In: Okudaira M, Mizumoto $\mathrm{R}$, Tanigawa $\mathrm{H}$, editors. Toriatsukai kiyaku ni sotta shuyou kanbetsu shindan atlas kanzou. Tokyo: Bunkodo; 1991:22-27. Japanese. ${ }^{17}$

relatively larger in size but smaller in number in the $\mathrm{pHCC}$ state. In other words, the individual tumor sinusoids become larger during the process of $\mathrm{HCC}$ dedifferentiation. ${ }^{8}$ This affects the appearance of $\mathrm{HCC}$ on the portal venous phase (PVP) of dynamic CT or MRI because the intratumoral flow may simply follow the Hagen-Poiseuille Law. ${ }^{8}$ Therefore, the resistance would be higher when there are a large number of small sinusoids and lower when there are a small number of large sinusoids, leading to slower flow within wHCC and faster flow within $\mathrm{pHCC}$, respectively. ${ }^{8}$ Thus, $\mathrm{wHCC}$ tend to show persistent enhancement on PVP, whereas $\mathrm{mHCC}$ and pHCC tend to show so-called "washout" on PVP. ${ }^{8}$ A schematic presentation of this concept is shown in Figure 3.

\section{Outflow system}

The HCC outflow system has also been shown to undergo significant sequential change during multistep hepatocarcinogenesis. ${ }^{3,9,10}$ In dysplastic or early HCC nodules, it is thought that hepatic vein-like vessels remain within the nodules, resembling normal liver tissue. Therefore, the intratumoral blood flow, which originates from the portal venous flow rather than the hepatic arterial flow as mentioned earlier, drains into the intratumoral venous system. In wHCC nodules, before formation of a pseudocapsule, the intratumoral venous structures diminish in number or are obliterated due to a gradually elevated intratumoral pressure secondary to increased cellular density. The intratumoral flow, which is mainly of hepatic arterial origin, outflows via the sinusoids into the surrounding nontumorous liver tissue, because in the absence of a fibrous capsule, the tumor sinusoids are in direct contact with the surrounding nontumorous sinusoids (transsinusoidal pathway or drainage). This transient blood influx into the nontumorous liver tissue surrounding the HCC nodules causes thin ring-like enhancement on single-level dynamic CTHA (SLCTHA) or the late phase of CTHA. ${ }^{3,9,10}$ In wHCC to mHCC nodules, which form pseudocapsules, the intratumoral venous structure completely disappears due to elevated intratumoral pressure. ${ }^{3,9,10}$ Because the pseudocapsule serves as a barrier to trans-sinusoidal drainage, the intratumoral blood flow, which is entirely of hepatic arterial origin, drains out of the tumors via small residual portal venous branches located within the pseudocapsule into the nontumoral liver tissue around the lesion, and subsequently, into the hepatic venous system. ${ }^{3,9,10}$ This transient blood influx into the nontumorous liver tissue surrounding the HCC nodules causes irregularly shaped, thick enhancement, known as "corona-like enhancement", around the tumor on SLCTHA or the late phase of CTHA. ${ }^{3,9,10}$ In moderately to poorly differentiated HCC nodules, which have a disrupted or absent pseudocapsule, the drainage route of the intratumoral blood flow is a mixture of the trans-sinusoidal pathway and a partial "corona-like enhancement". A schematic presentation of this concept is shown in Figure 4, and actual representative cases are shown in Figure 5.
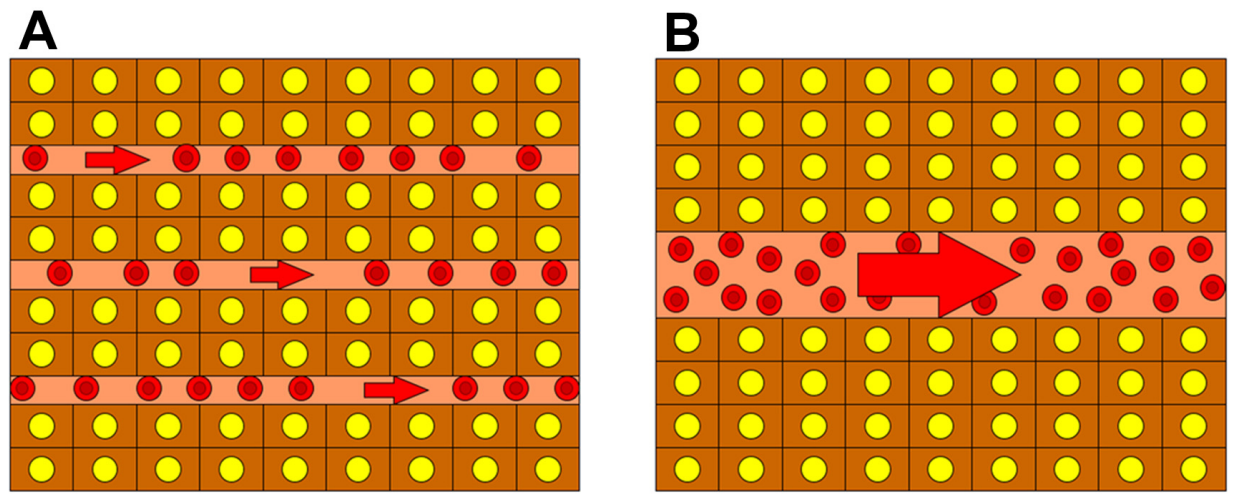

Figure 3 Schematic presentation of the sequential changes to the tumor sinusoidal structure during multistep hepatocarcinogenesis. (A) Well-differentiated hepatocellular carcinoma with a large number of thin sinusoids present; (B) poorly differentiated hepatocellular carcinoma with a relatively small number of large sinusoids present. Note that the total area of the tumor sinusoids is the same as that of $(\mathbf{A})$. 

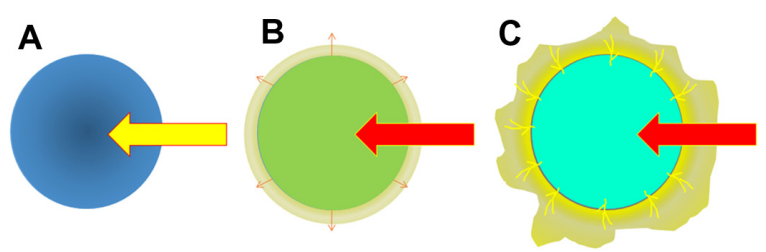

Figure 4 Schematic presentation of the venous drainage of hepatocellular neoplasms during multistep hepatocarcinogenesis.

Notes: (A) Hypovascular dysplastic nodules or early hepatocellular carcinoma. Because venous blood drains out of the lesions via vein-like vessels that connect to the venous system of the surrounding nontumorous tissue, there is no "corona-like enhancement" around the lesion. (B) Hypervascular well-differentiated hepatocellular carcinoma without pseudocapsule. Vein-like vessels decrease in number most likely due to elevated intratumoral pressure caused by increased cellularity. At the periphery of the lesion, the tumor typically shows a "replacing growth pattern", in which the tumor sinusoids directly face the sinusoids of the surrounding nontumorous hepatocytes. Venous blood from the lesions drains into the surrounding liver tissue via the "trans-sinusoidal pathway", which can be radiologically observed as a "thin ring-like enhancement" or "thin corona enhancement" on the second phase of CT during hepatic arteriography. An actual case corresponding to this is shown in Figure $5(A-C)$. (C) Hypervascular moderately to poorly differentiated hepatocellular carcinoma with pseudocapsular formation. Because of the presence of a pseudocapsule, sinusoids of the tumor and the surrounding liver tissue do not have direct contact in these lesions. Venous blood drains out of the lesions via residual portal venous branches present within the pseudocapsule. These portal venous branches drain into the sinusoids of the surrounding liver tissue, and subsequently, into the venous system, which can be radiologically observed as a typical "corona-like enhancement" on the second phase of CT during hepatic arteriography.An actual case corresponding to this is shown in Figure 5 (D-F).

Abbreviation: CT, computed tomography.

\section{Consideration of Lp-TACE based on structural and hemodynamic changes during multistep hepatocarcinogenesis}

Considering the structural and hemodynamic changes of HCC during multistep hepatocarcinogenesis as mentioned above, it is reasonable that the therapeutic effects of Lp-TACE may be affected during this process. First, HCC nodules that are purely and abundantly supplied by arterial blood flow may be more susceptible to intra-arterially administered chemotherapeutic agents or embolic materials. In addition, lesions with anaerobic metabolism, which is characteristic of pHCC, should be resistant to ischemia caused by embolic therapy. mHCC, therefore, would be the most sensitive to TACE or Lp-TACE in this regard. Second, the size and number of HCC tumor sinusoids may influence the effects of chemotherapeutic agents. According to previous observations, ${ }^{14,15}$ a Lipiodol emulsion/suspension can pass through the tumor sinusoids and reach the portal aspect of the HCC. Assuming that Lipiodol emulsions/suspensions fill the sinusoidal space completely, the total area of HCC cells that are in direct contact with a Lipiodol emulsion/suspension is larger in HCC consisting of small tumor sinusoids than in those with larger tumor sinusoids, assuming the ratios of sinusoidal areas are the same (Figure 6). Therefore, tumor cells in wHCC or mHCC may have a greater chance of being exposed to chemotherapeutic agents than those in pHCC. Third, it is thought that the more established the outflow system is, the greater the embolic effect of TACE or Lp-TACE will be in terms of both arterial and portal venous side blockage. Thus, mHCC or pHCC may be more effectively treated by TACE or Lp-TACE than wHCC in this regard.

Considering all the above-mentioned factors, $\mathrm{mHCC}$, which is the most commonly encountered histological grade of HCC in clinical practice, is the grade that is most effectively treated by TACE or Lp-TACE (Figure 7). Furthermore, some mHCC may be able to be controlled by simple ischemia

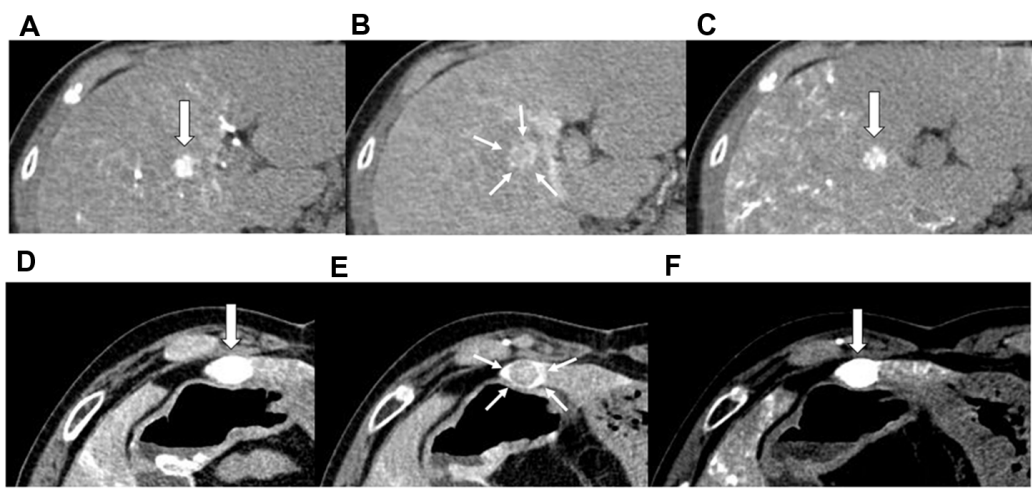

Figure 5 Two examples of venous drainage patterns of hypervascular hepatocellular carcinomas on CTHA. A 53-year-old man with chronic hepatitis C (A-C) and a 65-year-old woman with chronic hepatitis C (D-F).

Notes: (A) The first phase of CTHA of a small hepatocellular carcinoma without pseudocapsule formation. The main body of the tumor is strongly enhanced (arrow). (B) The second phase of CTHA demonstrates thin ring-like enhancement around the lesion, indicating venous drainage of the tumor (small arrows). (C) Unenhanced CT obtained immediately after LP-TACE. Note that Lipiodol is sporadically present only within the main body of the tumor (arrow) and does not cover the ring-like enhancement as shown in (B). The tumor recurred I month later. (D) The first phase of CTHA of a larger hepatocellular carcinoma with pseudocapsule formation. The main body of the tumor along with drainage area ("corona-like enhancement") is strongly enhanced (arrow). (E) The second phase of CTHA demonstrates washout of the main body of the lesion and irregularly shaped "corona-like enhancement" around the lesion, indicating venous drainage of the tumor (small arrows). (F) Unenhanced CT obtained immediately after LP-TACE. Note that Lipiodol densely covers almost the entire area of corona-enhancement and the main body of the lesion (arrow), as shown in (D) and (E). The tumor showed complete response in I month.

Abbreviations: CT, computed tomography; CTHA, CT during hepatic arteriography; LP-TACE, transarterial chemoembolization using iodized oil. 

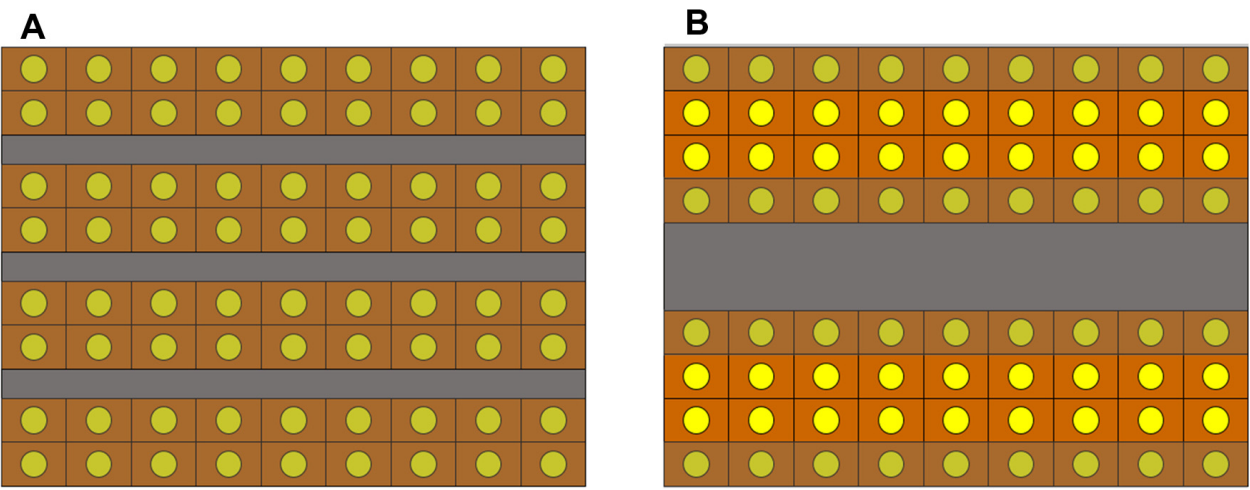

Figure 6 Schematic presentation of the relationship between the distribution of Lipidol emulsion/suspension and histological grades of hepatocellular carcinomas. Notes: (A) Well or moderately differentiated hepatocellular carcinoma. Because the total contact area of the tumor cells to Lipiodol is large, the chemotherapeutic agent may affect all of the tumor cells. Tumor cells that may be influenced by chemotherapeutic agents are shown in dark color. (B) Poorly differentiated hepatocellular carcinoma. Although the total area of tumor sinusoids is the same as that of $(\mathbf{A})$, the contact areas between Lipiodol and the tumor cells are smaller in (B) compared to (A). Tumor cells that are located in the central part of the thick tumor plates may be less influenced by chemotherapeutic agents (shown in lighter color), whereas those having direct contact with the tumor sinusoids are more likely to be affected by chemotherapeutic agents (shown in darker color).

caused by bland embolization without chemotherapeutic agents or simple chemo-lipiodol emulsion/suspension injection without embolic material administration. In contrast, for tumors that are more highly or poorly differentiated than typical mHCC, a well-balanced therapeutic approach may be required, particularly for $\mathrm{pHCC}$; this approach provides sufficient chemotherapeutic and ischemic effects. In most cases of HCC nodules, some intralesional heterogeneity in terms of histological grade exists; thus, this balanced approach is necessary to achieve consistently good control of HCC by means of Lp-TACE.

In Japan, epirubicin is the most frequently used chemotherapeutic agent for Lp-TACE, but cisplatin ${ }^{19}$ and miriplatin ${ }^{20}$ are also used as second-line treatment or as alternative agents. Although there is no evidence to suggest which agent is the best suited for TACE at present, considering the repetitive

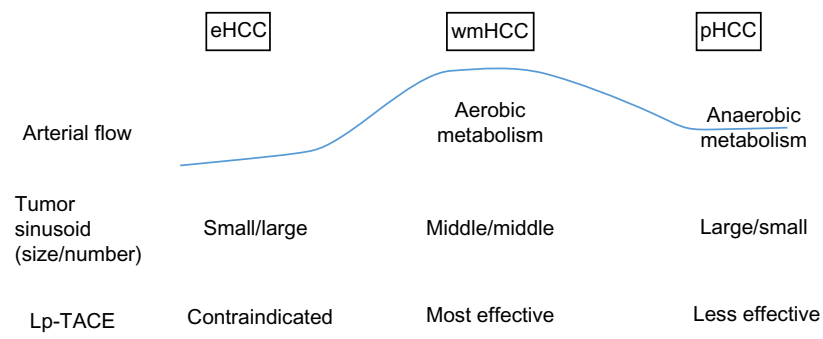

Figure 7 Summary of hemodynamic and structural changes during multistep hepatocarcinogenesis and their relationship to LP-TACE.

Notes: Because of rich arterial supply, aerobic metabolism, abundant contact areas between tumor cells and chemotherapeutic agents, and a complete venous drainage system, moderately differentiated hepatocellular carcinoma may be most effectively controlled by TACE. To treat poorly differentiated hepatocellular carcinoma, both robust chemotherapeutic and sufficient ischemic effects may be required.

Abbreviations: eHCC, early HCC; HCC, hepatocellular carcinomas; LP-TACE, transarterial chemoembolization using iodized oil; $\mathrm{pHCC}$, poorly differentiated HCC; TACE, transarterial chemoembolization; wmHCC, well to moderately differentiated HCC. nature of TACE procedures, it is important to use the agent that will not only provide the greatest antitumor effect but also minimize damage to nontumorous liver tissue to preserve liver function after TACE for any future procedures. ${ }^{21}$

\section{Conclusion}

From the perspective of the sequential changes to inflow hemodynamics, sinusoidal structures, and outflow hemodynamics (venous drainage) during multistep hepatocarcinogenesis, the therapeutic effect of Lp-TACE should be greatest for mHCC, which is the most common histological type encountered in clinical practice. To control pHCC, both robust chemotherapeutic and sufficient ischemic effects are necessary. Considering the histological heterogeneity within $\mathrm{HCC}$ nodules, the ideal Lp-TACE treatment would strike a good balance between chemotherapeutic and ischemic effects, while causing the least possible damage to nontumorous liver tissue.

\section{Disclosure}

KY has supervised the development of educational material for Dainippon Sumitomo Pharma Co, Ltd. The author reports no other conflicts of interest.

\section{References}

1. Matsui O, Kadoya M, Kameyama T, et al. Benign and malignant nodules in cirrhotic livers: distinction based on blood supply. Radiology. 1991;178(2):493-497.

2. Ueda K, Terada T, Nakanuma Y, Matsui O. Vascular supply in adenomatous hyperplasia of the liver and hepatocellular carcinoma: a morphometric study. Hum Pathol. 1992;23(6):619-626.

3. Matsui O, Kobayashi S, Sanada J, et al. Hepatocelluar nodules in liver cirrhosis: hemodynamic evaluation (angiography-assisted CT) with special reference to multi-step hepatocarcinogenesis. Abdom Imaging 2011;36(3):264-272. 
4. Hayashi M, Matsui O, Ueda K, et al. Correlation between the blood supply and grade of malignancy of hepatocellular nodules associated with liver cirrhosis: evaluation by CT during intraarterial injection of contrast medium. AJR Am J Roentgenol. 1999;172(4):969-976.

5. Tajima T, Honda H, Taguchi K, et al. Sequential hemodynamic change in hepatocellular carcinoma and dysplastic nodules: CT angiography and pathologic correlation. AJR Am J Roentgenol. 2002;178(4):885-897.

6. Asayama Y, Yoshimitsu K, Irie H, et al. Poorly versus moderately differentiated hepatocellular carcinoma: vascularity assessment by computed tomographic hepatic angiography in correlation with histologically counted number of unpaired arteries. J Comput Assist Tomogr. 2007;31(2):188-192.

7. Asayama Y, Yoshimitsu K, Nishihara Y, et al. Arterial blood supply of hepatocellular carcinoma and histologic grading: radiologic-pathologic correlation. AJR Am J Roentgenol. 2008;190(1):W28-W34.

8. Okamoto D, Yoshimitsu K, Nishie A, et al. Enhancement pattern analysis of hypervascular hepatocellular carcinoma on dynamic MR imaging with histopathological correlation: validity of portal phase imaging for predicting tumor grade. Eur J Radiol. 2012;81(6):1116-1121.

9. Ueda K, Matsui O, Kawamori Y, et al. Hypervascular hepatocellular carcinoma: evaluation of hemodynamics with dynamic CT during hepatic arteriography. Radiology. 1998;206(1):161-166.

10. Kitao A, Zen Y, Matsui O, Gabata T, Nakanuma Y. Hepatocarcinogenesis: multistep changes of drainage vessels at CT during arterial portography and hepatic arteriography-radiologic-pathologic correlation. Radiology. 2009;252(2):605-614.

11. Higashihara H, Okazaki M. Transcatheter arterial embolization of hepatocellular carcinoma: a Japanese experience. Hepatogastroenterology. 2002;49(43):72-78.

12. Matsui O, Miyayama S, Sanada J, et al. Interventional oncology: new options for interstitial treatments and intravascular approaches: superselective TACE using iodized oil for HCC: rationale, technique and outcome. J Hepatobiliary Pancreat Sci. 2010;17(4):407-409.

13. Ikeda M, Arai Y, Park SJ, et al; Japan Interventional Radiology in Oncology Study Group (JIVROSG); Korea Interventional Radiology in Oncology Study Group (KIVROSG). Prospective study of transcatheter arterial chemoembolization for unresectable hepatocellular carcinoma: an Asian cooperative study between Japan and Korea. J Vasc Intervent Radiol. 2013;24(4):490-500.
14. Terayama N, Matsui O, Gabata T, et al. Accumulation of iodized oil within the nonneoplastic liver adjacent to hepatocellular carcinoma via the drainage routes of the tumor after transcatheter arterial embolization. Cardiovasc Intervent Radiol. 2001;24(6):383-387.

15. Miyayama S, Matsui O, Yamashiro M, et al. Ultraselective transcatheter arterial chemoembolization with a 2-f tip microcatheter for small hepatocellular carcinomas: relationship between local tumor recurrence and visualization of the portal veins with iodized oil. J Vasc Intervent Radiol. 2007;18(3):365-376.

16. Ikeda K, Kobayashi M, Saitoh S, et al. Origin of neovascular structure in an early stage of hepatocellular carcinoma: study of alpha-smooth muscle actin immunohistochemistry in serial thin sections of surgically resected cancer. J Gastroenterol Hepatol. 2006; 21(1 Pt 1):183-190.

17. Okudaira M. II kansaibougan 2. soshiki bunrui [Hepatocellular carcinoma 2. Histological classification]. In: Okudaira M, Mizumoto R, Tanigawa H, editors. Toriatsukai kiyaku ni sotta shuyou kanbetsu shindan atlas kanzou. Tokyo: Bunkodo; 1991:22-27. Japanese.

18. The Liver Cancer Study Group of Japan. The General Rules for the Clinical and Pathological Study of Primary Liver Cancer. 5th ed. Tokyo: Kanahara; 2008:38-45.

19. Maeda N, Osuga K, Higashihara H, et al. Transarterial chemoembolization with cisplatin as second-line treatment for hepatocellular carcinoma unresponsive to chemoembolization with epirubicin-Lipiodol emulsion. Cardiovasc Intervent Radiol. 2012;35(1):82-89.

20. Kora S, Urakawa H, Mitsufuji T, Osame A, Higashihara H, Yoshimitsu K. Warming effect on miriplatin-lipiodol suspension as a chemotherapeutic agent for transarterial chemoembolization for hepatocellular carcinoma: preliminary clinical experience. Cardiovasc Intervent Radiol. 2013;36(4):1023-1029.

21. Iwazawa J, Hashimoto N, Ohue S, Muramoto O, Mitani T. Chemoembolization-induced arterial damage: evaluation of three different chemotherapeutic protocols using epirubicin and miriplatin. Hepatol Res. 2014;44(2):201-208.
Hepatic Medicine: Evidence and Research

\section{Publish your work in this journal}

Hepatic Medicine: Evidence and Research is an international, peerreviewed, open access journal covering all aspects of adult and pediatric hepatology in the clinic and laboratory including the following topics: Pathology, pathophysiology of hepatic disease; Investigation and treatment of hepatic disease; Pharmacology of drugs used for

\section{Dovepress}

the treatment of hepatic disease. Issues of patient safety and quality of care will also be considered. The manuscript management system is completely online and includes a very quick and fair peer-review system, which is all easy to use. Visit http://www.dovepress.com/ testimonials.php to read real quotes from published authors. 